\title{
INITIAL STRESS CORRECTION METHOD FOR THE MODELING OF FOLDED SPACE INFLATABLE STRUCTURES
}

\author{
Yanan ZHAN ${ }^{1}$, Li YU ${ }^{2}$, Xue YANG ${ }^{3}$, Han CHENG ${ }^{4}$
}

College of Aerospace Engineering, Nanjing University of Aeronautics and Astronautics, Nanjing China

E-mail: ${ }^{1}$ zhanyanan03@163.com; ${ }^{2} y u l i$ happy@163.com (corresponding author);

31287245258@qq.com; ${ }^{4}$ chenghanstorm@sina.com

Received 24 March 2014; accepted 10 October 2014

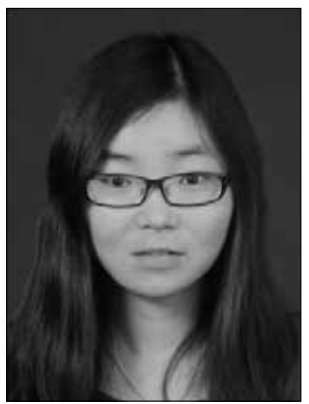

Yanan ZHAN, MSc student

Date of Birth: 1990.

Education: 2009 to 2013, Undergraduate student. 2013 to present, master student. Both at the College of Aerospace Engineering, Nanjing University of Aeronautics and Astronautics. Research interest: the dynamics of space inflatable structures.

Publications: author of 1 scientific article and co-author of 2 scientific articles.

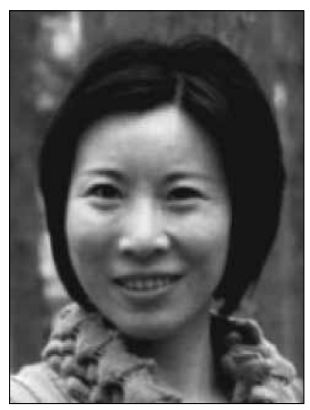

Li YU, Prof.

Date of Birth: 1969.

Education: Nanjing University of Aeronautics and Astronautics. 2006, PhD (Eng).

Affiliations and functions: member of Safety and Rescue Committee in CSAA; member of Return and Re-entry Committee in CSA; editor of "Space Recovery \& Remote Sensing".

Research interest: fluid structure interaction; ADS design; environmental control systems. Publications: over 30 research papers.

Present position: professor and doctoral tutor at the College of Aerospace Engineering, Nanjing University of Aeronautics and Astronautics.

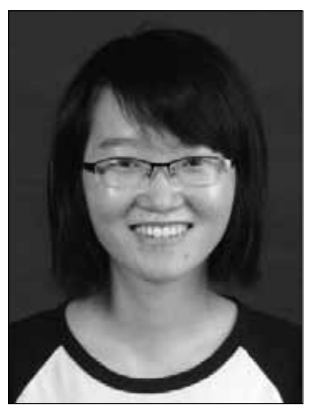

Xue YANG, MSc student

Date of birth: 1991.

Education: 2009-2013, undergraduate student. 2013 to present: master student. Both at the College of Aerospace Engineering, Nanjing University of Aeronautics and Astronautics. Research interest: immersed boundary theory.

Publications: co-author of 2 scientific articles.

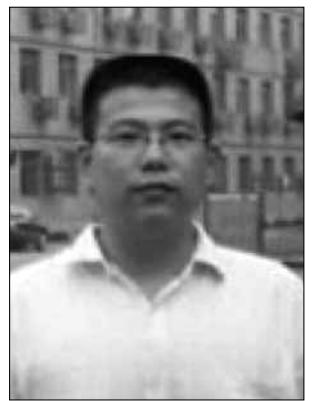

Han CHENG, PhD student

Date of birth: 1984.

Education: 2003-2007, undergraduate at Changzhou University; 2007-2010, master student, 2010-2014, PhD student, both at the College of Aerospace Engineering, Nanjing University of Aeronautics and Astronautics.

Present position: instructor at the Civil Aviation Flight University of China.

Research interest: fluid structure interaction; ADM design.

Publications: over 10 research papers. 
Abstract. Initial errors and mesh distortions are inevitable in the modelling of folded space inflatable structures. Aimed at this key technical problem, an initial stress correction method based on finite element theory is proposed in this paper. First, initial stress is calculated through the difference of mapping and reference configurations, the former with initial errors and the later without. Then the initial stress is imposed on the mapping configuration to correct initial errors. Through the correction, the accuracy of the inflation deployment numerical calculation is greatly improved. In order to validate the reliability of this correction method, a typical space inflatable structure-inflatable tube is studied as an example. First of all, the finite element models of both Z-folded and rolled tubes are established with the initial stress correction method. Then the inflation deployment numerical calculations of the folded tubes are carried out applying the segmented inflatable control volume method. Through comparative analysis of the calculation results with and without a correction, the method proposed in this paper is proved to be feasible and accurate. The correction method is a complement to the modelling of folded space inflatable structures and it has a great significance for the improvement of the accuracy of the inflation deployment numerical calculations of space inflatable structures.

Keywords: space inflatable structure, initial error, initial stress correction method, inflation deployment numerical calculation.

\section{Introduction}

Inflatable structures have a series of advantages such as small package volume, light weight, low cost and high deployment reliability. Therefore, they have become a new form of space structure that is widely accepted in various space missions, such as solar arrays, sunshades, solar concentrators, space habitation and antennas (Cassapakis, Thomas 1995; Freeland et al. 1998). In the deployment process, some problems exist, such as nonlinear contact between adjacent walls of a folded tube, material nonlinearity of tubes, long time of deployment process, low inflation rate, inner pressure non-uniform in the tubes, large deformation and displacement, etc. (Guan et al. 2009; Wei 2008). Undoubtedly, all these have a direct impact on the deployment dynamics of space inflatable structures, the precision of deployment and the accuracy of structure surface. An unexpected or unsuccessful deployment can induce a great waste of manpower, physics and financial resources. It is difficult for ground tests to forecast its deployment process in space, since the simulation of a huge spatial working environment is limited by gravity and air. Therefore, it is not easy to understand the deployment mechanics of space inflatable structures.

However, with the rapid development of computer hardware, numerical calculation has become an important research method due to its short calculation cycle, low resource consumption and high precision results. At the same time, the foldability of inflatable structures becomes a major difficulty for numerical modeling that has to be solved at first. Therefore, a variety of modeling methods has been proposed by scholars, such as general folded modeling, direct folded modeling, initial matrix modeling, etc. (Zhang et al. 2005; Wan, Yang 2005; Tanavde et al. 1995). Nevertheless, with the increasing complexity of the shape and folded pattern of inflatable structures, initial errors and mesh distortions generated by traditional modeling methods seriously affect the accuracy of numerical calculations. In order to respond to this increasingly significant problem, partial and over- all correction methods have been proposed (Tan et al. 2010; Ma et al. 2004). In the partial correction method, wrinkles are introduced in the inner layer to ensure that the inside and outside wall lengths are similarly equal. It is obvious that the operation is difficult and the workload is large. What is more, it causes quality deterioration of the local elements as well as introduces new individual errors. In the overall correction method, the length of the elongated layers is multiplied by a scaling factor in the elongate direction to offset the length increase due to folding. Though this correction method is very easy, it requires that the length difference of the inner and outer layers is small. Also, $\mathrm{t}$ is usually applied in the case of the correction of two layer rolled folding. As the above correction methods are essentially based on a geometric distortion and do not follow any conservation law, it is natural that the correction effect is limited for complex structures.

When confronted with the difficulties and limitations of the existing numerical modeling and correction methods, the initial stress correction method is proposed in this paper. Since this method is based on mechanics, and a complex geometric transformation matrix is avoided, accuracy can be improved. Moreover, the inflation deployment numerical calculations of Z-folded and rolled tubes are taken as examples to validate the reliability of this method. Through contrastive analysis of the calculation results with and without a correction, this method is proved to be obviously effective as well as easily operated. It is of a great significance for improving the precision of inflation deployment numerical calculations of space inflatable structures.

\section{Numerical model errors}

Initial errors and mesh distortions are introduced inevitably during the folding process as existing modeling methods are entirely based on geometric distortion and the conversion does not follow any conservation law. Usually initial errors are neglected when a structure is simple or only a few layers are folded. But for the folding 
of many layers or one that demands a high precision, such as for solar sails (Cadogan et al. 1999), initial errors and element distortions cannot be ignored.

In order to analyse the initial errors of folded models, the flat and rolled folding commonly used in engineering are studied as objects. The area of the inflatable structure is $4 \times 4 \mathrm{~m}$ and the interval between the upper and lower layers is $0.02 \mathrm{~m}$. Shell elements with four nodes are applied for the walls of the membrane and the element size is $0.2 \mathrm{~m}$. A flat direct folded model based on inner layers is established (Fig. 1). During the folding process, the elements of the outer layer are stretched by $10 \%$ and the inner elements are almost unchanged. While in the unfolded area, the element size of $0.2 \mathrm{~m}$ is retained. When the mesh model increases to 5 layers, the initial errors will reach $45.3 \%$. For a rolled folding with 5 layers (Fig. 2), the initial error is $15.7 \%$, with almost all elements affected.

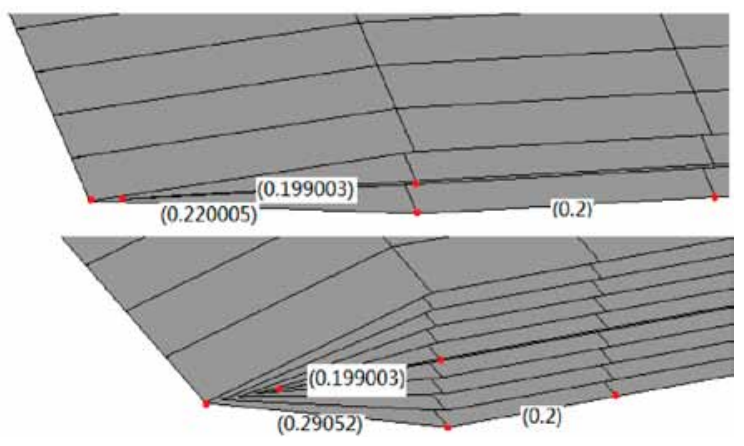

Fig. 1. Initial errors of flat direct folding

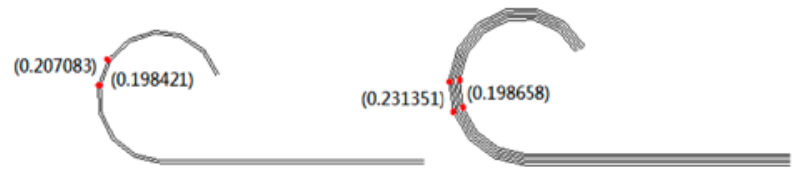

Fig. 2. Initial errors of rolled folding

\section{Mathematical model}

\subsection{Initial stress correction theory}

The initial stress correction method is based on finite element theory, which is different from the existing method based on geometric principles. The correction theory is shown below (Fig. 3).

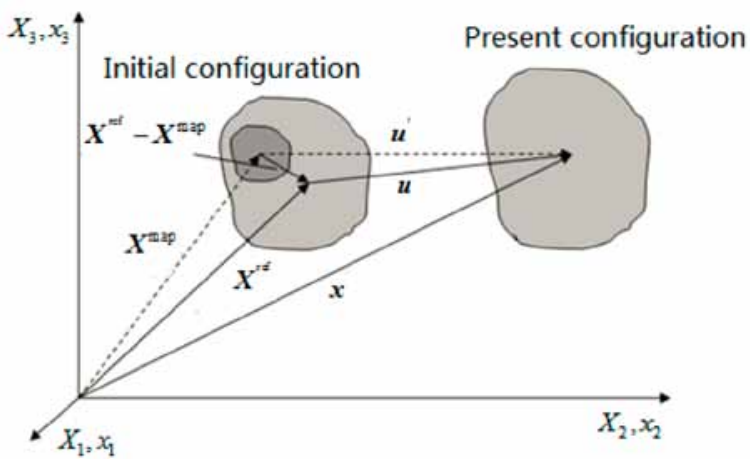

Fig. 3. Replacement of the initial configuration
In figure 3, $X^{\text {map }}$ and $X^{\text {ref }}$ represent the coordinates of meshes with and without initial errors respectively. The former is used as the mapping configuration, and the later as the reference one. The Green Stress $E$ is obtained by calculating the difference between mapping and reference configurations $X^{r e f}-X^{m a p}$. Then correction stress $S$ is calculated based on the material constitutive equation:

$$
S=C_{i j k l} E,
$$

where $S$ is the second Piola-Kirchhoff stress and $\mathrm{C}_{i j k l}$ is the fourth-order tangent modulus tensor.

The actual correction process is illustrated below (Fig. 4).

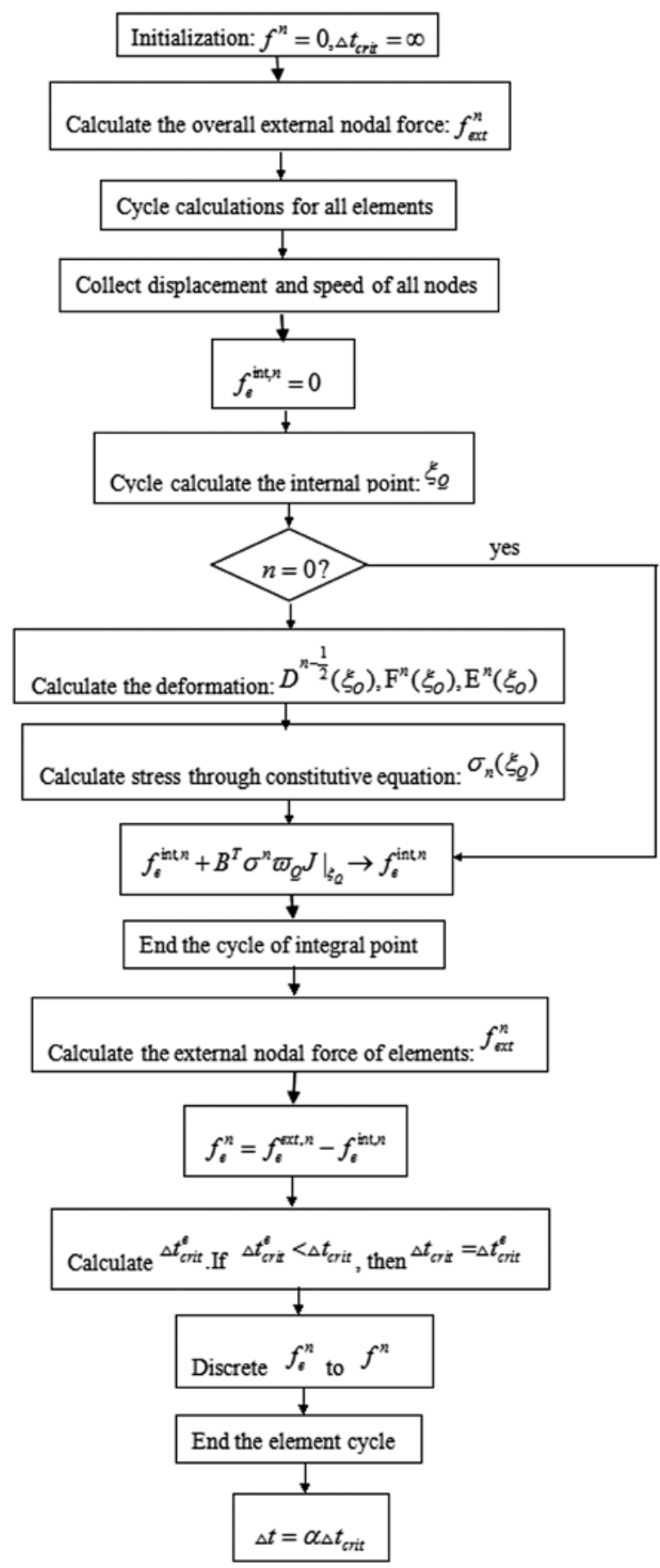

Fig. 4. Steps of initial stress correction 
In figure $4 f^{n}$ indicates the force of step n; $\Delta t_{\text {crit }}-$ the critical step; $f_{\text {ext }}^{n}$ - the external force of step n; $f_{e}^{\text {int }, n}$ - internal force of elements in step n; $\xi_{\underline{o}}$ - the integration point. $D^{n-\frac{1}{2}}$ is the deformation rate of step $n-\frac{1}{2} ; F^{n}$ - deformation gradient; $E^{n}$ - green strain tensor; $\sigma_{n}$ - cauchy stress; $B^{T}$ - geometric matrix; $\Phi_{o}$ - integral weight; $J$ - Jacobian determinant; $\Delta f_{\text {crit }}^{e}$ - the critical time step of elements; $\alpha$ - adjustment coefficient.

In explicit finite element calculations, the replacement of initial configuration can be realized when correction stress is applied to the mapping configuration at a certain time $t$. Hence, the initial numerical model with errors can be corrected.

\subsection{Specific realization of correction}

The specific realization of initial stress correction on applications is illustrated below (Fig. 5).

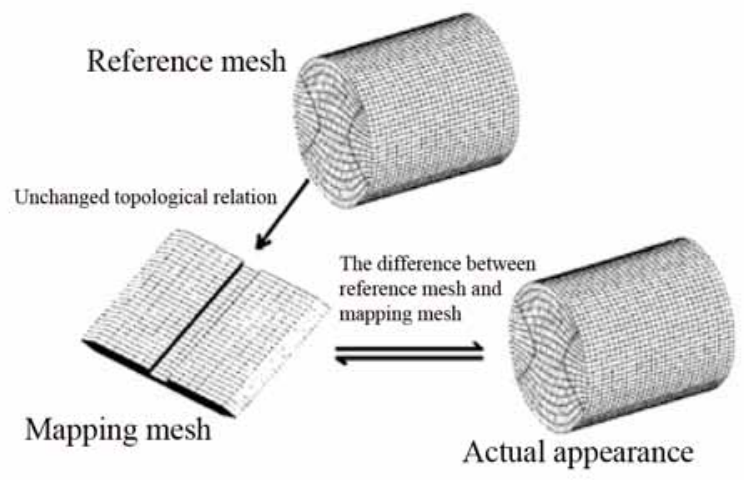

Fig. 5. Specific realization of correction

The finite element model which is fully expanded or without initial errors is selected as the reference mesh. Then the reference model is folded through a transformation matrix $\boldsymbol{T}$.

$$
\begin{gathered}
{\left[\begin{array}{llll}
x_{1}^{*} & x_{2}^{*} & x_{3}^{*} & 1
\end{array}\right]=\left[\begin{array}{llll}
x_{1} & x_{2} & x_{3} & 1
\end{array}\right] \cdot T=} \\
{\left[\begin{array}{lllll}
x_{1} & x_{2} & x_{3} & 1
\end{array}\right] \cdot\left[\begin{array}{llll}
a_{11} & a_{12} & a_{13} & a_{14} \\
a_{21} & a_{22} & a_{23} & a_{24} \\
a_{31} & a_{32} & a_{33} & a_{34} \\
a_{41} & a_{42} & a_{43} & a_{44}
\end{array}\right],}
\end{gathered}
$$

where $\left[\begin{array}{llll}x_{1} & x_{2} & x_{3} & 1\end{array}\right]$ and $\left[\begin{array}{llll}x_{1}^{*} & x_{2}^{*} & x_{3}^{*} & 1\end{array}\right]$ are the homogeneous coordinates of elements before and after folding, respectively. The transformation matrix $\left[\begin{array}{llll}a_{11} & a_{12} & a_{13} & a_{14} \\ a_{21} & a_{22} & a_{23} & a_{24} \\ a_{31} & a_{32} & a_{33} & a_{34} \\ a_{41} & a_{42} & a_{43} & a_{44}\end{array}\right]$ can be divided into four sub matrixes. $\left[\begin{array}{lll}a_{11} & a_{12} & a_{13} \\ a_{21} & a_{22} & a_{23} \\ a_{31} & a_{32} & a_{33}\end{array}\right]$ can realize three types of geometric transformations, namely scaling, rotation and shearing. $\left[\begin{array}{lll}a_{41} & a_{42} & a_{43}\end{array}\right]$ can realize translation. $\left[\begin{array}{lll}a_{14} & a_{24} & a_{34}\end{array}\right]^{T}$ can realize projection and $\left[a_{44}\right]$ can realize overall scaling.

Despite the fact that many initial errors and mesh distortions are introduced into the folding process, the topology relationship remains unchanged. Naturally, the models before and after folding can be respectively used as reference and mapping configurations. The difference of the mapping and reference configurations is calculated as the initial displacement to modify the mapping configuration. Then a deployed structure which matches the actual shape is obtained.

The Segmented inflatable control volume method (SICV) used in the numerical calculations of inflation deployment is presented in the following references (Wei 2008; Salama et al. 1999; Wang, Jonson 2002).

\section{Finite element model of an inflatable tube}

First of all, the geometric model of an inflatable tube in the flattening state is built up by adopting modeling ideas from the bottom to the top. The length of the cylindrical fabric tube is $320 \mathrm{~mm}$, the diameter is $60 \mathrm{~mm}$ and the wall thickness is $0.1 \mathrm{~mm}$. The material is considered as an isotropic elastic material. The wall of the membrane tube adopts a shell element with four nodes, the size of which is $0.004 \mathrm{~m}$. In order to simulate the progressive deployment process, the closed interconnected tube is divided into four control volumes, respectively named $C V_{1}, C V_{2}, C V_{3}$ and $C V_{4}$. The control volumes are separated by three orifices (the orifices are set in the fold line). The flattening model is shown in (Fig. 6). In the numerical calculations of inflation, the $C V_{1}$ initially provides gas pressure, and the other three control volumes get pressure from the former through the orifice. The mass flow rate is $0.0015 \mathrm{~g} / \mathrm{s}$.

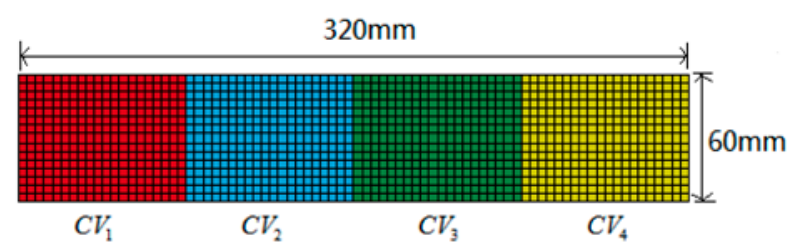

Fig. 6. The mesh model in the flattening state

The Z-folded and rolled tube models are established accordingly from the flattening model (Fig. 6). For the Z-folded model (Fig. 7), the inlet boundary $\mathrm{AB}$ is fixed: $u=v=w=0$, and the node $\mathrm{C}$ and $\mathrm{D}$ in the top have a displacement boundary condition: $u=v=0$. For the rolled model (Fig. 8), the displacement boundary condition is also applied and the inlet boundary EF is fixed: $u=v=w=0$. 


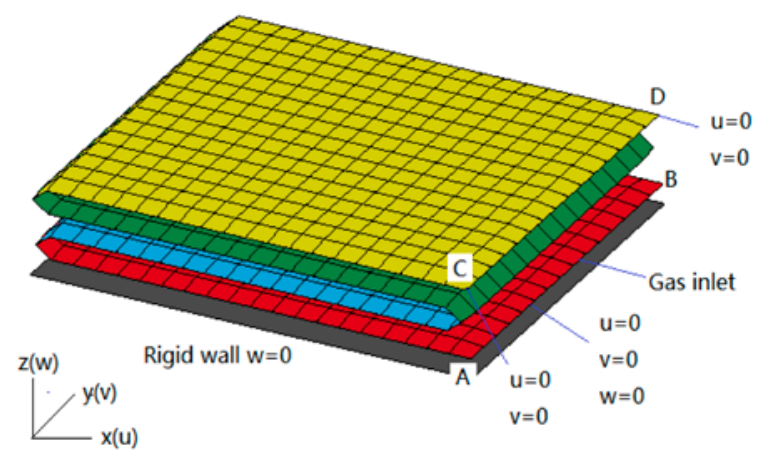

Fig. 7. Finite element model of a Z-folded tube

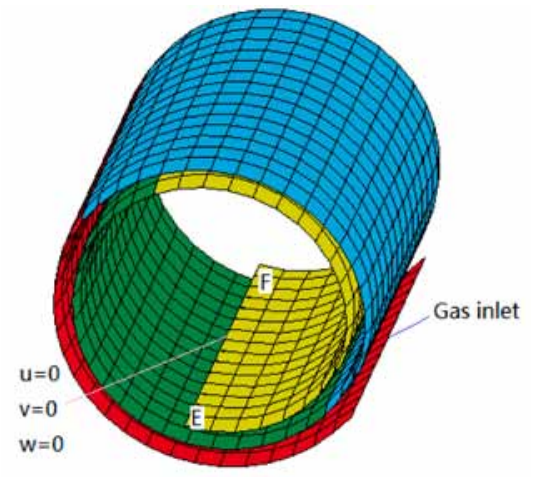

Fig. 8. Finite element model of a rolled tube

As in the second section of the analysis, the initial errors and mesh distortions of the folded models are inevitable. But the serial number and the order of both the nodes and elements remain unchanged and the topological relationship remains the same. Therefore, the finite element model of the flattening of the tube in figure 6 can be directly used as the reference configuration, and the folded models in figures 7 and 8 can be used as the mapping ones. Then the initial stress correction method is applied to the mapping configurations for corrections. The correction effect is shown in (Fig. 9). For the Z-folded model, the initial difference of the mapping and reference configurations is in the fold line, as the folded meshes are affected by initial stress. While for the rolled model, the elements in the deformation domain all have varying degrees of changes; hence, all elements are affected.
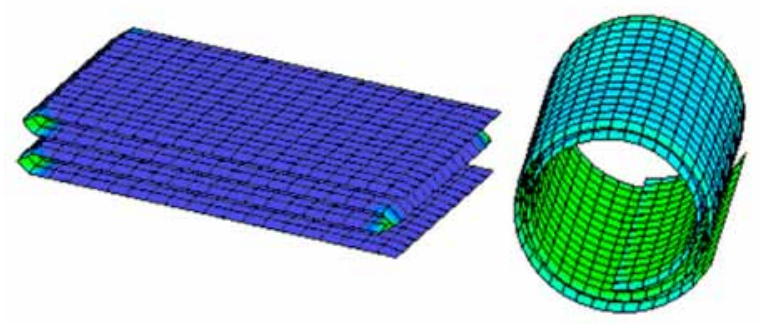

Fig. 9. Effect of initial stress correction

\section{Comparative analysis of the calculation results}

The uncorrected $\mathrm{Z}$-folded model is referred to as model $\mathrm{A}$, and the corrected one is referred to as model B. Similarly, the uncorrected rolled model is referred to as model $\mathrm{C}$, and the corrected one is referred to as model D. The numerical calculations of inflation deployment are carried out by applying the Segmented Inflation Control Volume method (SICV) for the four models.

\subsection{Comparative analysis of the Z-folded model}

Equivalent stress distributions of model A and B in the deployment process are shown in (Fig. 10) to illustrate the effect of initial errors, which also proves the reliability and accuracy of the correction method. From the point of the deployment configuration, the calculation results are consistent with the numerical results in the paper (Wang, Jonson 2002) and the experimental results in the paper (Hill, Jacob 2010). The process before $0.2 \mathrm{~s}$ is referred to as the inflation stage. At this stage, the appearance of model A and B is almost the same, and stress concentration appears in distorted and constrained elements. After $\mathrm{t}=0.2 \mathrm{~s}$, the correction effect becomes obvious. For model A, the folds and wrinkles are distinct, and the stress concentration always exists even when the fold has been expanded. When in full deployment, the appearance is not consistent with the actual state. While for the corrected model $\mathrm{B}$, there is no mesh distortion, and the stress concentration and the wrinkle both disappear when the fold is fully deployed. After the inflation is finished, the appearance is completely smooth, agreeing well with the actual state.

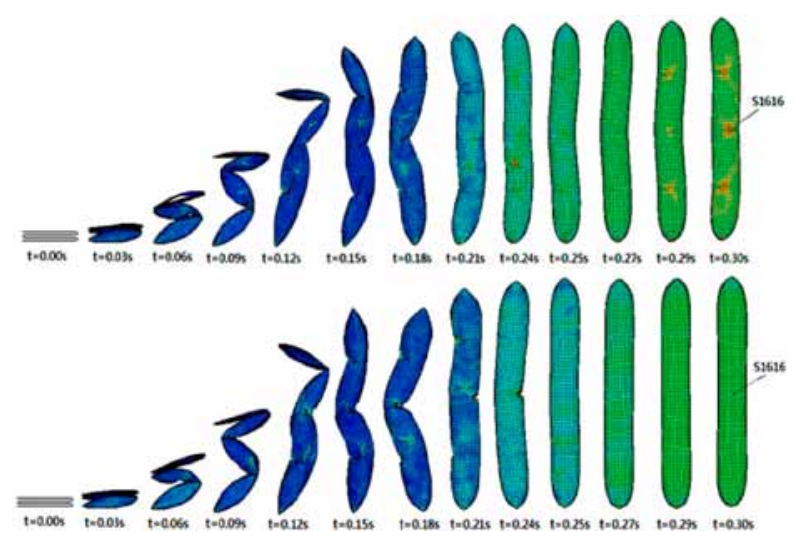

Fig. 10. Equivalent stress distributions in the deployment process of a $\mathrm{Z}$-folded model. The top model is model A; the lower is model B

The equivalent stress variation of element 1616 during the deployment process is presented in figure 11 (the position of the element is shown in figure 10). During the inflation stage, the equivalent stresses of model $\mathrm{A}$ and $\mathrm{B}$ are almost the same. However, after $\mathrm{t}=$ 
$0.25 \mathrm{~s}$, the equivalent stress of model B is always smaller than that of model A, which can account for the result that the stress concentration of model A always exists while the model B's disappeared in the later inflation stage shown in figure 10.

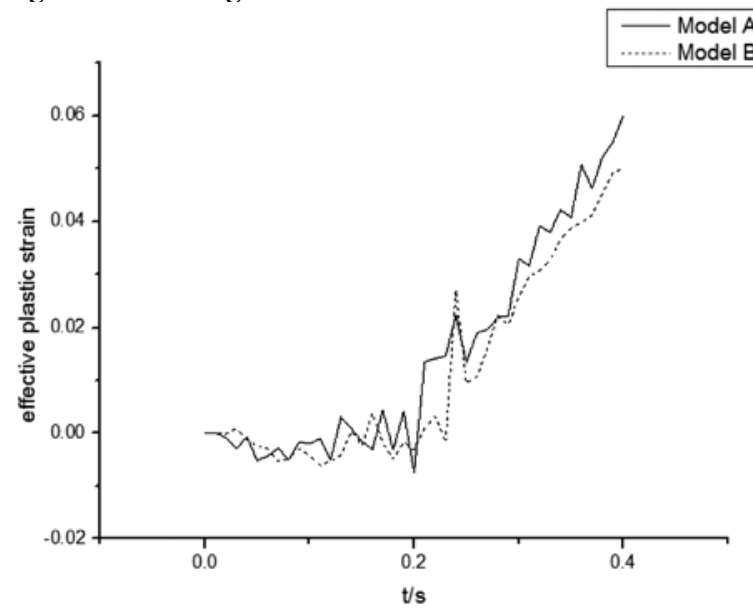

Fig. 11. Equivalent stress variation of the element in the fold

The element size contrast of the distorted element with and without correction is shown in (Fig. 12). For the uncorrected model A, the element error of the outer layer is $9 \%$ and the inner layer is $-23.8 \%$. For the corrected model $\mathrm{B}$, the element error of the inner and outer layer is $0.75 \%$ and $0.25 \%$, respectively. It is evident that the accuracy is greatly increased.

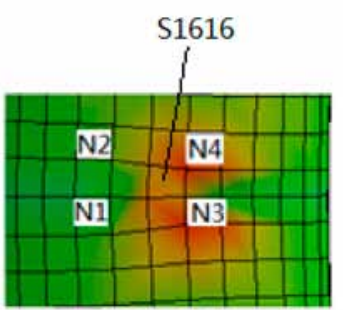

$\mathrm{N} 1 \mathrm{~N} 2=0,00436 \mathrm{~m}$ $\mathrm{N} 3 \mathrm{~N} 4=0.00305 \mathrm{~m}$

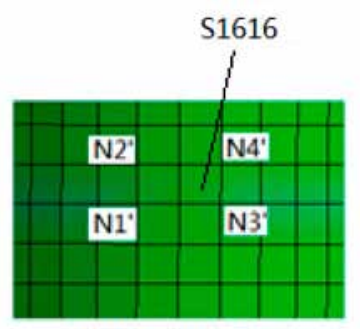

$\mathrm{N} 1^{\prime} \mathrm{N} 2{ }^{\prime}=0.00403 \mathrm{~m}$ $\mathrm{N} 3^{\prime} \mathrm{N}^{\prime}=0.00401 \mathrm{~m}$
Fig. 12. Error analysis of the element size. Model A is on the left and model $B$ is on the right

The error analysis of the tube's axial size after inflation is shown in (Fig. 13). The reference model is inflated and the axial size of the deployed reference model is $0.3114 \mathrm{~m}$, which can be regarded as the standard size. For the uncorrected model A, the axial size is $0.3052 \mathrm{~m}$, with an error of $-2 \%$. Whereas for the corrected model B, the axial size is $0.3115 \mathrm{~m}$ and the error is just $0.03 \%$. It is obvious that the precision is greatly improved. What regards the appearance of deployed tubes, the fact is that the corrected one is consistent with the reference one, while the uncorrected is not, which proves that the initial stress correction method is efficient.
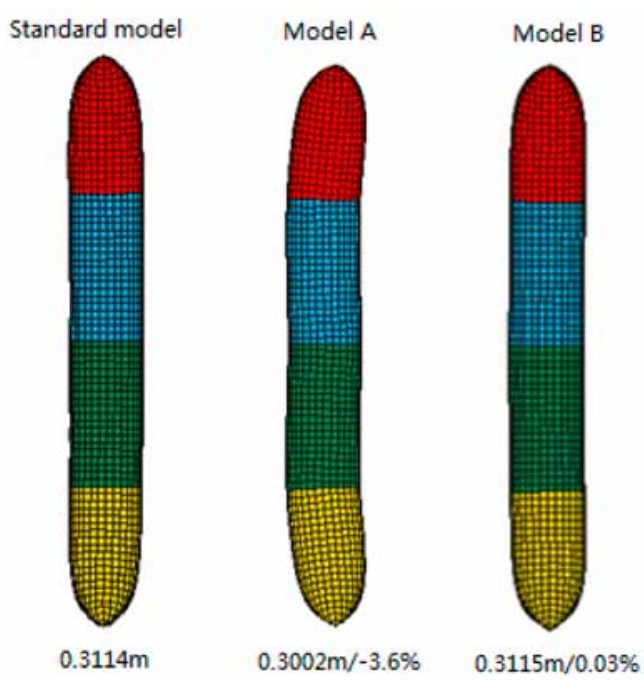

Fig. 13. Error analysis of the axial size after deployment

\subsection{Comparative analysis of a rolled model}

The equivalent stress distributions of model C and D in the deployment process are presented in (Fig. 14). From the point of the deployment configuration, the calculation results are consistent with the numerical results presented in (Wang, Jonson 2002) and the experimental results given in (Hill, Jacob 2010). In the inflation stage, the stress concentration appears in the fold. But as the fold is expanded, both the wrinkles and the stress concentration disappear. After the inflation is finished, for the uncorrected model $\mathrm{C}$, the track length of the inner layer is always smaller than that of the outer layer, so the tube appears to be at a bending state, which does not agree with the actual state. Whereas for the corrected model $\mathrm{D}$, the track lengths of the inner and outer layer keep similar equality, and the appearance is actually full and smooth, and consistent with the actual state.

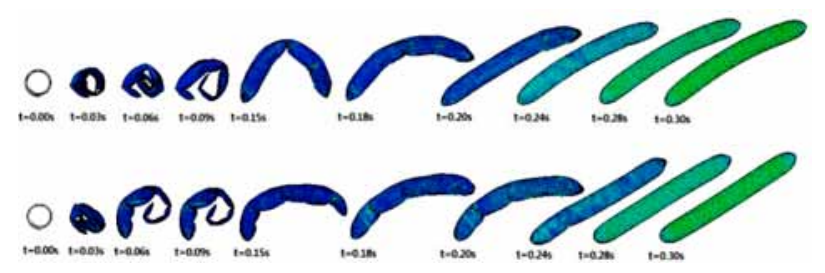

Fig. 14. Equivalent stress distortion in the inflation process of a rolled model. Model C is at the top while model D is at the bottom

The element size contrast of the inner and outer mesh with and without correction is shown in (Fig. 15). For the size of the inner element, models $\mathrm{C}$ and $\mathrm{D}$ both remain almost unchanged. However, for the outer size, model C has an error of $5.9 \%$, while the error of model D is just $0.35 \%$. It is obvious that the accuracy is greatly improved. 


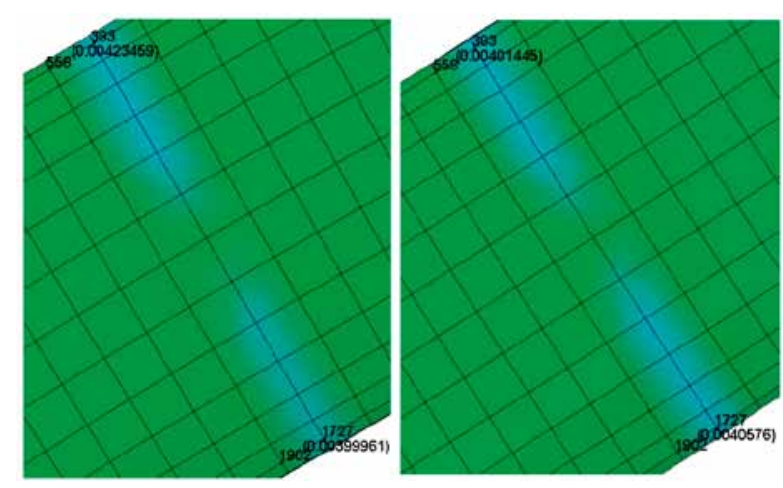

Fig. 15. Error analysis of the element size. Model C is on the left while model $\mathrm{D}$ is on the right

\section{Conclusions}

The inevitable initial errors and mesh distortions generated by the modeling of folded space inflatable structures will with no doubt affect the accuracy of numerical calculations of inflation deployment. Aimed at this key technical problem, the initial stress correction method is proposed in this paper. Also, the inflation deployment numerical calculations of $\mathrm{Z}$-folded and rolled tube models are taken as examples to validate the reliability and the accuracy of this method. The distinct advantages of the correction method can be summarized as follows:

1) the operation is easy while the effect is obvious. The accuracy of numerical calculations can be greatly improved by applying this correction method;

2) as long as the topological relationship remains unchanged in the modeling process, the model before folding can be used as the reference configuration and the folded one can be used as the mapping configuration. Therefore, the mapping relationship can be easily established;

3) essentially, the initial stress correction method is based on finite element theory, so it is not influenced by the complexity of modeling, and it can be applied to either complex structures or structures demanding a high precision.

The correction method proposed in this paper is a complement for the modeling of folded space inflatable structures. It is significant for improving the precision of the inflation deployment numerical calculations of space inflatable structures.

\section{Acknowledgements}

The authors are grateful for the support of the National Natural Science Foundation of China (No. 11172737) and the Aeronautics Science Foundation of China (No. 20122910001).

\section{References}

Cadogan, D. P.; Lin, J. K., et al. 1999. Inflatable solar array technology, in $37^{\text {th }}$ AIAA Aerospace Science Meeting and Exhibit, 11-14 January 1999, Reno, NV. AIAA-99-1075[online], [cited 10 March 2014]. Available from Internet: http://arc.aiaa.org/doi/pdf/10.2514/6.1999-1075

Cassapakis, C.; Thomas, M. 1995. Inflatable structures technology development overview, in Proceedings of AIAA Space Programms and Technologies Conference, 26-28 September 1995, Huntsville, Ala. USA, American Inst. of Aeronautics and Astronautics Inc. AIAA-95-3738 [online], [cited 10 March 2014]. Available from Internet: http://www.lgarde. com/assets/content/files/publications/overview.pdf

Freeland, R. E.; Bilyeu, G. D.; Mikulas, M. M. 1998. Inflatable deployable space structures technology summery, in $49^{\text {th }}$ International Astronautical Congress, 28 September - 02 October 1998, Melbourne, Australia. IAF98-I.5.01, 1-16 [online], [cited 10 March 2014]. Available from Internet: http://d.wanfangdata.com.cn/ExternalResource-zrht200904006\%5E1.aspx

Guan, F. L.; Xu, Y.; Zhen, Y. 2009. Applications of inflatable structures technology for space exploration, Manned Space Flight 15(4): 40-48 [online], [cited 10 March 2014]. Available from Internet: http://d.wanfangdata.com.cn/periodical_zrht200904006.aspx

Hill, J.; Jacob, J. 2010. Deployment of inflatable space habitat models, in $48^{\text {th }}$ AIAA Aerospace Sciences Meeting Including the New Horizons Forum and Aerospace Exposition, 4-7 January 2010, Orlando, Florida. AIAA-2010-793 [online], [cited 10 March 2014]. Available from Internet: http://enu. kz/repository/2010/AIAA-2010-793.pdf

Ma, C. S.; Huang, S. L., et al. 2004. Analysis and adjustment of geometric errors in modeling airbag folding processes, Journal of Tsinghua University (Science \& Technology) 44(11): 1540-1543 [online], [cited 10 March 2014]. Available from Internet: http://d.wanfangdata.com.cn/periodical_qhdxxb200411029.aspx

Salama, M.; Kuo, C. P., et al. 2000. Simulation of deployment dynamics of inflatable structures, AIAA Journal 38(12): 2277-2283 [online], [cited 10 March 2014]. Available from Internet: http://citeseerx.ist.psu.edu/viewdoc/download?doi=10.1.1.2.7757\&rep=rep1\&type $=$ pdf http://dx.doi.org/10.2514/2.896

Tanavde, A. S.; Khandelwal, H., et al. 1995. Airbag Modeling Using Initial Metric Methodology. The Engineering Society for Advancing Mobility Land Sea Air and Space [online], [cited 10 March 2014]. Available from Internet: http://papers.sae.org/950875/

Tan, D. W.; Li, L., et al. 2010. Modeling modification and deployment simulation of inflatable tube with typical folded configurations, Journal of Astronautics 31(11): 2610-2617 [online], [cited 10 March 2014]. Available from Internet: http://d.wanfangdata.com.cn/periodical_yhxb201011027. aspx

Wang, J. T.; Johnson, A. R. 2002. Deployment simulation methods for ultra-lightweight inflatable structures, in $43^{\text {rd }}$ AIAA/ ASME/ASCE/AHS/ASC/Structures, Structural Dynamics, and Material Conference. AIAA-99-1521 [online], [cited 10 March 2014]. Available from Internet: http://citeseerx.ist. psu.edu/viewdoc/download?doi=10.1.1.74.8088\&rep=rep1\&type $=$ pdf 
Wan, X. M.; Yang, J. K. 2005. The Simulation of the impact of the folding way on deployment force, Chinese Journal of Mechanical Engineering 41(12): 162-166 [online], [cited 10 March 2014]. Available from Internet: http://www. cnki.com.cn/Article/CJFDTotal-JXXB200512037.htm http://dx.doi.org/10.3901/JME.2005.12.162

Wei, J. Z. 2008. Research on Gas-Solid Interaction Problem in the Inflatable Deployment Process of Space Folded Membrane Boom: Dissertation. Harbin Institute of Technology [online], [cited 10 March 2014]. Available from Internet: http://cdmd.cnki.com.cn/Article/CDMD-102132009223840.htm

Zhang, J. H.; Ma, C. S.; Bai, Y. L. 2005. Airbag mapped mesh auto-flattening method, Tsinghua Science and Technology 10(3): 387-390 [online], [cited 10 March 2014]. Available from Internet: http://ieeexplore.ieee.org/stamp/stamp. jsp?arnumber $=06076051$

http://dx.doi.org/10.1016/S1007-0214(05)70086-9 